\title{
Модель межмолекулярного взаимодействия общего типа между молекулой и жидкой фазой, основанная на теории обобщенных зарядов
}

\author{
(C) 2020 Долгоносов А.М. \\ Институт геохимии и аналитической химии им. В.И. Вернадского РАН, Москва
}

Поступила в редакцию 19.03.2020 г.

DOI: $10.17308 /$ sorpchrom.2020.20/2871

В рамках проблемы моделирования методов высокоэффективной хроматографии предлагаемая статья посвящена обоснованию тех аспектов теории межмолекулярного взаимодействия, которые важны для описания селективности хроматографических неподвижных фаз и, в конечном счете, для априорного расчета хроматографического удерживания. Дается краткое изложение теории обобщенных зарядов, ее приложения для межатомных связей (гомополярная и донорно-акцепторная связи) и межмолекулярных сил (неэмпирическое представление потенциала Леннард-Джонса). Приводится описание водородной связи как связи гидридов без электронного обмена и соответствующего разделения зарядов. Каждый раздел теории прошел проверку экспериментальными данными, результаты которой приведены в литературных ссылках и частично представлены в статье.

Энергия межмолекулярного взаимодействия согласно предложенной модели содержит три независимые группы величин, описывающих неполярные, полярные силы и водородные связи. Каждая из сил представлена своим молекулярным дескриптором - соответственно обобщенным зарядом, дипольным моментом и двумя числами, отражающими способность молекулы быть донором или акцептором водородной связи. С помощью теории обобщенных зарядов получены связи «структура свойство» для всех составляющих энергии межмолекулярного взаимодействия. В частности, описан вклад водородной связи в общую энергию межмолекулярного взаимодействия как произведение пороговой величины, имеющей квантово-механическую природу, и вероятности правильного расположения взаимодействующих молекул. Указанная вероятность определяется как вероятность попадания системы взаимодействующих молекул в узкий потенциальный колодец и выражается через параметры молекулярной структуры. Развитая модель успешно применена для априорной оценки теплоты парообразования воды, спиртов и кислот, что послужило проверке разработанного подхода.

Ключевые слова: межмолекулярные взаимодействия, водородная связь, энергия адсорбции, теплота парообразования, обобщенный заряд.

\section{Введение}

Задача моделирования хроматографии заключается в создании компьютерных программ, способных по химическому составу пробы выбрать оптимальные условия разделения смеси, включая выбор состава подвижной и неподвижной фаз, режима разделения, предсказать результаты хроматографического эксперимента. Такие программы смогут решать и обратные задачи моделирования: проводить параметризацию модели и осуществлять теоретический анализ экспериментальных данных, необходимый для повышения достоверности химического анализа.

Разработанная в лаборатории сорбционных методов ГЕОХИ РАН программа IONCHROM [1] для моделирования ионной хроматографии решает указанные задачи и, в частности, способна провести классификацию весьма сложных ионообменных фаз фирмы DIONEX, являющихся наиболее эффективными и селективными 
среди существующих коммерческих фаз [2]. Высокий уровень моделирования с помощью IONCHROM позволил открыть новый режим градиентного элюирования (который можно назвать «линзой» за способность локального повышения разрешающей способности хроматографии) и разработать методику его применения [3]. Такие возможности имеются только у моделей высшего уровня, базирующихся на фундаментальных связях химической природы аналитов и их хроматографического поведения, на удачных и удобных представлениях исходных данных. Эти проблемы, успешно решаемые для ионной хроматографии, многократно усложняются для методов молекулярной хроматографии из-за многообразия аналитов и комплексного характера межмолекулярных взаимодействий (MMB), лежащих в основе сил межфазного распределения.

Между тем, современные методы молекулярного моделирования имеют целью не уточнение потенциала взаимодействия, а симуляцию поведения многочастичных систем [4]. Разработанные на их базе компьютерные программы служат целям наглядности и доступности расчетов, но не вносят в теорию межмолекулярных взаимодействий ничего нового. Более того, эти методы во многом противодействуют развитию фундаментальной теории, объявляя в силу своей грубости несущественными различия в применяемых потенциалах, т.е. отвергая необходимость более глубокого изучения MMB [5]. Однако имеется ряд проблем, которые вносят путаницу в важнейшие химические понятия. Это касается представлений о Н-связях, о потенциалах ван-дер-ваальсовых взаимодействий и др. [4-6]. Это сказывается, в частности, на результатах выбора равновесных конформаций сложных молекул, их конфигурации в группе и т.п.

Цель этой статьи обосновать модель MМВ, лежащую в основе классификации хроматографических фаз. Ранее на базе развиваемой автором теории обобщенных зарядов (ТО3) [7] был создан подход с выводом из первых принципов всех соотношений для априорного расчета адсорбции неполярных молекул на неполярных фазах, точности которого хватает на описание и обоснование разделения стереоизомеров сложных молекул на графите [8]. Здесь делается попытка распространить такой подход на широкий круг молекул и сорбентов.

\section{Краткое изложение теории обобщенных зарядов}

Квантово-статистическую теорию Томаса-Ферми $[9,10]$, на которой базируется современная теория функционала плотности $[10,11]$, удалось расширить на модель электронного газа с 4-мя компонентами, что привело к количественным соотношениям для атомных параметров и межатомных сил [12]. Ниже приведены параметры тетрадной модели электронного газа и выводимые из нее выражения TO3. Здесь для упрощения записи используются атомные единицы в рамках системы СГСЭ: $\hbar=e=m=1$, где $\hbar$ - приведенная постоянная Планка, $e, m-$ заряд и масса электрона, соответственно. Переход к привычным единицам: 1 ат. ед. длины=0.05292 нм - радиус Бора, 1 ат. ед. энергии=2625 кДж/моль - энергия Хартри.

В книге [12] предложена модель многокомпонентного электронного газа, расширяющая представления теории Томаса-Ферми $[9,10]$ на парные состояния в неоднородном электронном газе. Описываются 4 состояния-компоненты: синглет, триплет, неполный синглет и неполный триплет, - обозначенные индексами $i=0 \ldots 3$, соответственно; в этой связи для модели лучше подходит название «тетрадная модель». Первая компонента имеет свойства плотного газа Томаса-Ферми, а последняя 
— разреженного газа Мотта [10,13]. В тетрадной модели определяются доли компонент в электронной плотности:

$$
\gamma_{i}=\frac{\sqrt{2}-1}{3} \cdot 2^{(5-i) / 2}-\delta_{2 i}
$$

и в энергии атомного электронного газа:

$$
v_{i}=\frac{\sqrt{2}-1}{3} \cdot 2^{(5-i) / 2}-\delta_{2 i}-\delta_{3 i}
$$

где $\delta_{i j}=\left\{\begin{array}{l}1, i=j \\ 0, i \neq j\end{array}\right.$ - символ Кронекера $\left(v_{0 . .3}=0.781,0.552,-0.609,-0.724\right)$. Плотность электронного газа имеет только две независимые компоненты (в чистых состояниях - синглет и триплет), откуда следует, что 4-х-компонентное представление электронного газа имеет избыточный характер, и поэтому плотности четырех компонент связаны балансовыми соотношениями, в частности, в виде реакции

$$
e_{0}+e_{3} \Leftrightarrow e_{1}+e_{2}
$$

где $e_{i}$ обозначает компоненту электронного газа. Смысл реакции (3) - во взаимопревращении компонент при переносе электрона плотной компоненты синглета с индексом «0» или триплета «1» на вакансию дополнительной неплотной компоненты - соответственно неполного триплета «3» или неполного синглета «2»; тогда левая часть уравнения (3) переходит в правую и наоборот, в результате чего достигается равновесие.

Применение уравнения равновесия компонент дало взаимосвязи между парциальными плотностями в виде закона действия масс и, в конечном счете, квантовостатистическую связь между электронной плотностью $n_{i}(\mathbf{r})$ и потенциалом $\varphi(\mathbf{r})$ (измеряемым относительно потенциала точки нулевой плотности $\varphi_{0}$ ):

$$
n(\mathbf{r})=\frac{2 \sqrt{2}}{3 \pi^{2}}\left(\varphi(\mathbf{r})-\varphi_{0}\right)^{b},
$$

где $b=(9-i) / 6, i=0 \ldots 3$

Приложение полученных характеристик компонент к уравнению Пуассона, в которое подставлена плотность (4), приводит к уравнению самосогласованного по потенциалу поля для каждой компоненты, расширяющему уравнение Томаса - Ферми с показателем степени $b=3 / 2$ и на другие значения показателя (5):

$$
\frac{d^{2} \kappa}{d x^{2}}=x^{1-b} \kappa^{b}
$$

Нелинейное дифференциальное уравнение (6) является ключевым уравнением для тетрадной модели электронного газа, решения которого дают неэмпирическое описание атомных свойств и являются базовыми для теории обобщенных зарядов межатомных сил.

Граничные условия на атомном ядре и в точке нулевой электронной плотности имеют вид:

$$
\kappa(0)=1, \kappa\left(x_{0}\right)=0
$$

В случае нейтрального атома $x_{0}=\infty$; потенциал и радиальная координата определяются соотношениями

$$
\varphi(r)=\frac{A}{r} \kappa(B r)=\frac{A B}{x} \kappa(x), \quad x=B r,
$$

где $A, B$ - положительные постоянные, связанные благодаря граничному условию на ядре с зарядом ядра $Z$ : 


$$
A=Z, B=\left(\frac{8 \sqrt{2}}{3 \pi}\right)^{\frac{1}{3-b}} Z^{\frac{b-1}{3-b}}
$$

Потенциал электронного газа, равный взвешенной сумме потенциалов компонент, получающихся как решение уравнений (6), был использован для описания внутриатомного поля и таких свойств химических элементов, как полная энергия, энергия ионизации, размер атома и др. [14].

Свойство уравнения (6) описывать функцию, экспоненциально убывающую на небольших расстояниях, но сравнительно медленно (гиперболически) меняющуюся на больших расстояниях, позволяет рассмотреть полную величину в виде суммы двух полей: внутриатомного и межатомного. Для межатомного поля характерны две черты: оно подчиняется тем же уравнениям, что и внутриатомное поле, однако для него граничное условие на ядре несущественно. Исключение указанного условия приводит к новым константам поля вместо (9) и новому определению зарядов - так называемых, обобщенных зарядов.

В отсутствие граничного условия в начале координат (7) точное решение уравнения (6) для $i=0,1,1$ имеет вид:

$$
\begin{aligned}
& \kappa(a x) \stackrel{x>>1}{\longrightarrow}(x)=[2 c(2 c-1)]^{c} x^{1-2 c}, \\
& \text { где } c=\frac{1}{b-1}=\frac{6}{3-i}, \text { или } c_{0,1,2}=2,3,6,
\end{aligned}
$$

$a$ - масштабный множитель. Асимптотическая связь (10) отмечалась в $[9,10]$ для $b=3 / 2$. В точном решении уравнения (6) для компоненты неполного триплета ( $i=3$ ) нет гиперболической составляющей:

$$
\varsigma_{3}(x)=\kappa_{3}(a x)=\exp (-x)
$$

Безразмерная функция $\kappa(x)$ была определена в (8) как отношение потенциала поля электронного газа к кулоновскому потенциалу. Для обратной процедуры функции $\zeta(x)$ из (10) и (12) умножаются на «кулоновский» потенциал $Q / r$, определяемый зарядом нового поля $Q$, который назван обобщенным зарядом (O3). Потенциалы электронных компонент для удаленной от ядра области поля атома имеют вид:

$$
\begin{aligned}
& u(r)=\frac{B_{q} Q \varsigma(x)}{x}=\frac{A_{q} Q}{r^{2 c}}, i=0,1,2 \\
& u(r)=\frac{Q \exp \left(-B_{q} r\right)}{r}, i=3
\end{aligned}
$$

где $B_{q}=x / r, A_{q}=B_{q}{ }^{1-2 c}[2 c(2 c-1)]^{c}$. Указанные коэффициенты содержат множитель $\eta$ плотности О3, необходимый для ее нормировки: $\eta \int_{\Omega} n_{q} d \Omega=1$.

Одинаковая форма локальных соотношений для полей электронного газа и О3 реализуется для связи плотности $n_{q}$ и потенциала (ср. с (4)):

$$
n_{q}=\frac{2 \sqrt{2}}{3 \pi^{2}}\left(\frac{u}{Q}\right)^{b}
$$

Асимптотическому подобию внутриатомного и межатомного полей отвечает «водородная нормировка» - условие стыковки электростатического поля и поля О3 для атома водорода, внутриатомное поле которого вплоть до «орбиты» электрона $r=1$ имеет потенциал близкий к кулоновскому $1 / r$. Для атома водорода постулируется единичный О3. Равенство полей на орбите электрона, имеет вид: $A_{q(i=0)}=1$. Для 
определенного таким образом обобщенного заряда коэффициенты в $(13,14)$ принимают вид:

$$
A_{q}=\left[\frac{c(2 c+1)}{10}\right]^{c}, B_{q 3}=2 \sqrt{5}
$$

что соответствует константе

$$
\eta=15 \pi \sqrt{2} / 4
$$

и нормировке плотности О3 атома:

$$
4 \pi \int_{R_{i}}^{\infty} n_{q i}(r) r^{2} d r=\eta^{-1}=0.06002
$$

со значениями $R_{i}: 1.882,2.389,3.902$ и 0 - соответственно для $\boldsymbol{i}=0,1,2,3$.

С помощью $(13,14,16)$ в рамках одной компоненты записывается потенциал поля О3 и энергия взаимодействия двух О3 с индексами $j$ и $j$ ':

$$
\begin{gathered}
u=\left\{\begin{array}{l}
{[0.1 c(2 c+1)]^{c} Q r^{-2 c}, i=0,1,2} \\
Q r^{-1} \exp (-2 \sqrt{5} r), i=3
\end{array}\right. \\
U_{j j^{\prime}}=\left\{\begin{array}{c}
-[0.1 c(2 c+1)]^{c} Q_{j} Q_{j^{\prime}} r_{j j^{\prime}}^{-2 c}, i=0,1,2 \\
-Q_{j} Q_{j^{\prime}} r_{j j^{\prime}}^{-1} \exp \left(-2 \sqrt{5} r_{j j^{\prime}}\right), i=3
\end{array}\right.
\end{gathered}
$$

где коэффициенты из (16) для первых трех компонент при подстановке (11) равны: $1,9.261$ и $2.252 \cdot 10^{5}$, - соответственно.

Константа равновесия компонент в реакции (3), равная 1 для поля внутри атома, в случае межатомного поля зависит от расстояния:

$$
K_{q}(r) \equiv \frac{n_{q 0} n_{q 3}}{n_{q 1} n_{q 2}}=2.927 \cdot 10^{-8} r^{15} \exp (-4.472 r) .
$$

Функция $K_{q}(r)$ проходит через максимум $K_{q m}=6.852 \cdot 10^{-7}$ при значении аргумента $\mathrm{r}_{\mathrm{m}}=3.354$, а затем убывает до нуля. Таким образом, вне атома электронный газ расслаивается на пары компонент: ближе к атому, в области максимума пика, доминирует пара «синглет и неполный триплет» (с индексами «0» и «3»), дальше пара «триплет и неполный синглет» («1» и «2»). Фактически мы получили два независимых решения системы (20).

Учитывая локализацию, найдем, что первая пара компонент участвует в ковалентном взаимодействии, а вторая - в ван-дер-ваальсовом. В соответствии с $(2,20)$ для энергии ковалентного взаимодействия получим

$$
\begin{aligned}
& U=v_{0} U_{0}\left(r_{j j^{\prime}}\right)+v_{3} U_{3}\left(r_{j j^{\prime}}\right)= \\
& =-0.7810 Q_{0 j} Q_{0 j^{\prime}} r_{j j^{\prime}}^{-4}+0.7239 Q_{3 j} Q_{3 j^{\prime}} r_{j j^{\prime}}^{-1} \exp \left(-2 \sqrt{5} r_{j j^{\prime}}\right)^{\prime}
\end{aligned}
$$

а для энергии ван-дер-ваальсового взаимодействия - потенциал Леннард-Джонса:

$$
U=v_{1} U_{1}+v_{2} U_{2}=-5.115 Q_{1 j} Q_{1 j^{\prime}} r_{j j^{\prime}}^{-6}+1.373 \cdot 10^{5} Q_{2 j} Q_{2 j^{\prime}} r_{j j^{\prime}}^{-12}
$$

Вдали от ядра поле $\varphi$ электронного газа атома, имеющего электронную плотность $n_{e}$, с точностью до постоянного коэффициента совпадает с полем $u$ обобщенных зарядов, имеющим плотность $n_{q}$. Это условие в сочетании с $(15)$ приводит к пропорции:

$$
Q \propto\left(n_{e} / n_{q}\right)^{1 / b}
$$

Аддитивность плотности электронов, обобществленных в рамках жесткого фрагмента, составленного из группы более мелких фрагментов $j$, приводит к закону сложения обобщенных зарядов: 


$$
Q^{b}=\sum_{j} Q_{j}^{b}
$$

Эквивалентное (24) представление О3 имеет вид:

$$
Q=V^{1 / b}, \quad V=\sum V_{j}
$$

Из свойства (25) величины $V$, называемой электронным объемом, следует, что она линейно зависит от числа электронов, создающих поле ОЗ в рассматриваемом объеме системы, а водородная нормировка задает ее единицу как вклад $\sigma$-электрона.

Наконец, подстановка $(5,25)$ в формулу $(21)$ (в которой последний член в случае двухатомных молекул мал, и им можно пренебречь) даст выражение для энергии ковалентного взаимодействия:

$$
U \approx-0.7810 V_{j}^{2 / 3} V_{j^{\prime}}^{2 / 3} r_{j j^{\prime}}^{-4}
$$

Электронный объем (а с ним и обобщенный заряд) молекулярного фрагмента ограничен эффектом экранирования [12,15]. Как показано в [10], из модели ТомасаФерми в приближении локальной плотности следует выражение для радиуса координатной части сферической ячейки фазового пространства электрона - так называемого радиуса Вигнера-Зейтца. Радиус Вигнера-Зейтца является границей области распределения электрона в стационарном состоянии, его радиусом экранирования. В поле с потенциалом $E_{0} / z$ он равен:

$$
r_{s}=1.710 \sqrt{z / E_{0}}
$$

где $r_{s}$ - радиус экранирования электрона связи; $z$ - число электронов связи (удвоенная кратность связи), $E_{0}$ - модуль потенциальной энергии связи.

\section{Уравнение для энергии и длины ковалентной связи}

В работе [16] было получено уравнение

$$
x=\frac{0.8838}{\sqrt{y}} \cos ^{2 / 3}(0.6496 \sqrt{y})
$$

для безразмерных параметров длины $(x)$ и энергии $(y)$ гомополярной ковалентной связи:

$$
\begin{array}{r}
x=r_{e}\left(Z_{1} Z_{2}\right)^{-1 / 3}, \\
y=\frac{2}{z} E_{0} r_{e}^{2}=\frac{2}{z}\left(E r_{e}^{2}+\lambda\right)
\end{array}
$$

где $\lambda=\sum l(l+1) / 2$ - полуквадрат орбитального момента электронов связи в атомных единицах (ПКМ), $l$ - молекулярное орбитальное число, $r_{e}$ - длина связи, $E$ - энергия диссоциации связи: $E \approx E_{0}-\lambda r_{e}^{-2}$. Выражение для $E_{0}$ выводится в ТОЗ и имеет форму (26):

$$
E_{0} \approx \frac{4-2 \sqrt{2}}{3} z\left(Z_{1} Z_{2}\right)^{2 / 3} \frac{\alpha^{4 / 3}}{r_{e}^{4}}
$$

Здесь участвует так называемая функция связи, которая имеет смысл вероятности электронного обмена между атомами [12]:

$$
\alpha(r)=\sqrt{\frac{V_{1} V_{2}}{Z_{1} Z_{2}}}
$$

где $Z_{1}, Z_{2}$ - атомные номера связываемых элементов, $V_{1}, V_{2}$ - их электронные объемы. 
По определению, функция связи является интегралом перекрывания волновых функций валентных электронов, и ее вид приводится к косинусу полусуммы фаз электронов межатомной связи [17]:

$$
\alpha(r) \approx \cos \beta r, \beta=\frac{1}{2} \sum_{z} k_{i}
$$

где $k_{i}$ - проекция на ось связи волнового вектора электрона. Из условия неучастия экранированных электронов в образовании связи, $\alpha\left(r_{s}\right) \equiv 0$, коэффициент $\beta$ выражается через радиус экранирования:

$$
\beta=\frac{\pi}{2 r_{s}}
$$

Параметрами зависимости энергии диссоциации двухатомной молекулы от ее размера служат число $z$ и полуквадрат момента $\lambda$ электронов связи. Число электронов связи соответствует ее кратности (для простой связи 2 электрона и т.д.). Способ определения ПКМ связи базируется на анализе электронного строения связываемых атомов [16].

Уравнение (28) описывает универсальную закономерность, которая будет использована ниже. Для иллюстрации ее точности на рис.1 приведен средний участок зависимости $y(x)$ в сравнении с точками гомополярных связей, полученными в статье [16] из данных по двухатомным молекулам.

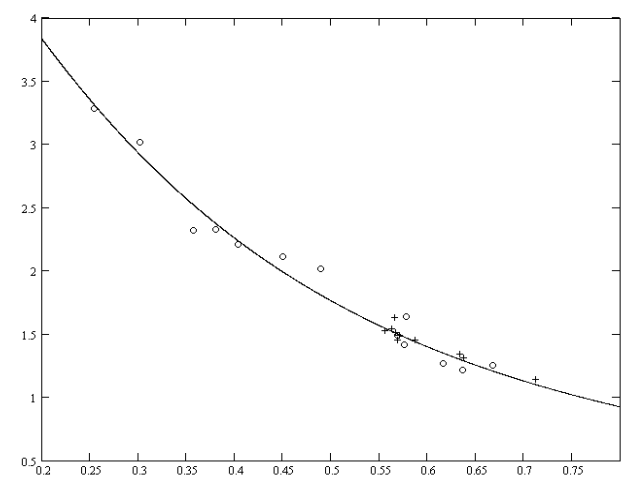

Рис. 1. Иллюстрация зависимости (28) для гомополярных молекул (сплошная линия). Обозначены экспериментальные точки: (о) - молекулы с простыми связями $\left(\mathrm{CCl}, \mathrm{CBr}, \mathrm{ClF}, \mathrm{BrF}, \mathrm{Cl}_{2}, \mathrm{Br}_{2}, \mathrm{JF}, \mathrm{BrJ}, \mathrm{J}_{2}, \mathrm{Au}_{2}, \mathrm{Hg}_{2}\right)$; (+) - молекулы с кратными связями $\left(\mathrm{C}_{2}, \mathrm{CN}, \mathrm{CO}, \mathrm{N}_{2}, \mathrm{NO}, \mathrm{O}_{2}, \mathrm{CS}, \mathrm{PO}, \mathrm{SO}, \mathrm{S}_{2}\right)$. Экспериментальные данные взяты из [18].

Совмещение теоретического графика с экспериментальными точками для двухатомных молекул (полученными из справочных данных [18]) говорит о хорошей точности теории ковалентной связи, основанной на ТОЗ. Для определения каждой из величин, связанных уравнением (28), требуются дополнительные сведения о характере связи. В этом ключе ниже рассмотрена разновидность полярного межатомного взаимодействия - донорно-акцепторная связь. Важным примером донорноакцепторных соединений для развиваемого здесь подхода являются соединения химических элементов с водородом - гидриды, при участии которых возникают водородные связи.

\section{Следствия теории для гидридов}

В работе [19] с позиций ТОЗ описан случай донорно-акцепторных связей, отличающийся от гомополярных связей тем, что в этом случае возникает состояние 
(с вероятностью $a=1-\alpha$ ), в котором $c$ электронов донора (его индекс «d») не возвращаются в свой атом, а оказываются локализованными в оболочке акцептора (индекс «a»). В этом состоянии атомы приобретают противоположные заряды величиной $c$. Энергия донорно-акцепторной связи является суммой вкладов каждого из атомов и кулоновской энергии зарядов за вычетом орбитального члена:

$$
E_{d a}=E_{d}+E_{a}+a \frac{c^{2}}{r_{e}}-\frac{\lambda}{r_{e}^{2}}
$$

Вклад в энергию атома-донора подчиняется выражению (31), записанному для одного из атомов, как если бы связь была гомополярной:

$$
E_{d}=\frac{1}{2} E_{0} \approx 0.3905 \frac{z}{2}\left(Z_{d} Z_{a}\right)^{2 / 3} \alpha^{4 / 3} r_{e}^{-4},
$$

где $z=2 c$. Энергия акцептора тратится на захват электронов, поэтому вкладом акцептора в энергию связи можно пренебречь $\left(E_{a} \approx 0\right)$. Таким образом:

$$
E_{d a} \approx 0.3905(z / 2)\left(Z_{d} Z_{a}\right)^{2 / 3} \alpha^{4 / 3} r_{e}^{-4}+(1-\alpha) c^{2} r_{e}^{-1}-\lambda r_{e}^{-2}
$$

В частном случае гидридов НХ получим при подстановке $\lambda=0, z=2 c=2$ и $Z_{d}=1$ :

$$
E_{\mathrm{HX}} \approx 0.3905 Z_{X}^{2 / 3} \alpha^{4 / 3} r_{e}^{-4}+(1-\alpha) r_{e}^{-1}
$$

Формула (38) хорошо согласуется с экспериментальными данными [18].

При захвате электрона акцептор становится отрицательным ионом. Такой ион можно представить как атом с характеристиками $Z+1$ (полное число электронов в ионе) и $V+1$ (число электронов, достигающих области взаимодействия). У водорода (протона) отношение $V / Z$ равно 1. Таким образом, для связи в гидридах согласно (32) имеем:

$$
\alpha_{\mathrm{HX}}=\sqrt{\frac{V+1}{Z+1}},
$$

где $Z, V$ - атомный номер и электронный объем элемента $\mathrm{X}$.

При использовании $(27,33,34,39)$ в работе [19] была выведена формула для длины связи гидридов:

$$
r_{\mathrm{HX}}=3.771 \arccos \sqrt{\frac{V+1}{Z+1}},
$$

и найдено выражение для электронного объема:

$$
V=Z-v_{n} ; v_{n}=2(n-1)^{2}-\sqrt{2}(n-2),
$$

где $Z, n-$ атомный номер и номер периода элемента $\mathrm{X}$.

Формулы (40) и (41) приводят к выражению, описывающему длины гидридов для широкого множества элементов:

$$
r_{\mathrm{HX}}=3.771 \arcsin \sqrt{\frac{v_{n}}{Z+1}}
$$

В работе [19] демонстрируется хорошее соответствие результатов расчета по формуле (42) экспериментальным значениям для соединений с водородом элементов 2-го - 6-го периодов. Итак, получены следствия, удовлетворительно описывающие длину и энергию гидридов аналитическими выражениями.

\section{Водородная связь - случай гидридов}

Метод описания гидридов удобен для перехода к слабой электронной связи Н-связи. В работе [20] делаются очевидные изменения в описании гидридов и с помощью универсального уравнения приходят к описанию Н-связи. 
Пусть, для определенности, водородная связь возникает между атомом водорода гидрида НY и атомом X соседней молекулы. В отличие от гидрида у водородной связи Н...X электрон водорода не может переходить к атому X, так как соответствующая орбиталь занята, поэтому выражение для энергии ограничивается первым членом в (38):

$$
E_{\mathrm{H} . . \mathrm{X}} \approx 0.3905 Z_{X}{ }^{2 / 3} \alpha^{4 / 3} r_{e}^{-4}
$$

Энергия в этой формуле относится к одному электрону - электрону водорода. Кроме того, выражение (43) имеет вид энергии гомополярной связи (31), т.е. с учетом формул $(29,30)$ подчиняется уравнению $(28)$ :

$$
E_{\mathrm{H} . \ldots \mathrm{X}}(x)=\frac{y(x)}{2 Z_{X}^{2 / 3} x^{2}},
$$

Таким образом, наряду с чертами донорно-акцепторной связи гидридов Нсвязь имеет характер одноэлектронной гомополярной связи.

В соответствии с выражением (32) имеет место равенство:

$$
\alpha_{\mathrm{H} . . \mathrm{X}} \approx \alpha_{\mathrm{HX}}
$$

Ввиду того, что Н-связь идет по оси гидрида, почти не отклоняясь, к ее характеристикам часто относят расстояние между атомами, связанными атомом водорода:

$$
r_{\mathrm{Y} \ldots \mathrm{X}} \approx r_{\mathrm{H} . . . \mathrm{X}}+r_{\mathrm{HY}}
$$

где $r_{\mathrm{HY}}-$ длина ковалентной связи гидрида.

Имея равные значения функции связи для одних и тех же элементов в Н-связи и гидриде, $\alpha_{\mathrm{H} . . \mathrm{x}} \approx \alpha_{\mathrm{HX}}$, эти связи осуществляются разными числами электронов (соответственно 1 и 2). Сумма проекций на межъядерную ось волновых векторов $\sum k_{i}$ в случае ковалентной связи вдвое больше проекции волнового вектора единственного электрона водородной связи. Из формулы (33): $\alpha=\cos \left(r \sum k_{i} / 2\right)$, следует соотношение между длинами гидридов и водородных связей для одних и тех же акцепторов:

$$
r_{\mathrm{H} . . \mathrm{X}} \approx 2 r_{\mathrm{HX}}
$$

\section{Нижний предел для энергии водородной связи}

Межатомные связи, осуществляемые валентными электронами, к которым относится и Н-связь, удовлетворяют условию финитности движения электронов: в области связи потенциальная энергия электрона по модулю не меньше его кинетической энергии, - в противном случае связь не образуется. Квантово-механическое ограничение на область распределения электрона водорода связывает размер этой области и его предельную кинетическую энергию при финитном движении.

Таким образом, искомое условие определяют следующие факторы [20]:

1) соотношение неопределенностей (в форме Вейля - для квадратичных приращений), где в качестве неопределенности положения электрона водорода рассмат-

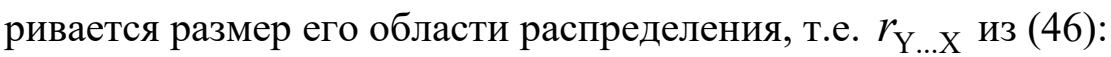

$$
r_{\mathrm{Y} \ldots \mathrm{X}} \delta p_{r} \geq 1 / 2
$$

где $\delta p_{r}$ - неопределенность модуля проекции импульса на ось связи;

2) в изотропном случае средний квадрат приращения полного импульса электрона втрое больше среднего квадрата приращения проекции импульса на какоелибо из трех направлений:

$$
(\delta p)^{2}=3\left(\delta p_{r}\right)^{2}
$$


3) кинетическая энергия электрона $t$ определяется из неравенства, связывающего квадрат величины с квадратом приращения ее модуля:

$$
t=p^{2} / 2 \geq(\delta p)^{2} / 2 ;
$$

4) условие финитного движения - кинетическая энергия не больше глубины потенциальной ямы:

$$
t \leq|U|
$$

5) согласно теореме вириала для замкнутой финитной электростатической системы, величины полной и потенциальной энергии относятся, как 1:2. Сделав необходимые подстановки, для модуля энергии электрона, осуществляющего Н-связь, получим: $E=\frac{|U|}{2} \geq \frac{t}{2} \geq \frac{3}{16} r_{\mathrm{Y} . . . \mathrm{X}}^{-2}$. В обозначениях формулы (46) рассматривается Нсвязь гидрида НY с атомом X, а так как энергия связи - это энергия ее электронов, то получим искомое условие:

$$
E_{\mathrm{H} \ldots \mathrm{X}} \geq \frac{3}{16} r_{\mathrm{Y} \ldots \mathrm{X}}^{-2}
$$

\section{Водородное связывание с учетом ограничений}

Для случая Н-связей в чистых веществах, представляющих собой однородную среду гидридов НX (т.е. когда $\mathrm{Y}=\mathrm{X})$, согласно (46) и (47) получим:

$$
r_{\mathrm{X} \ldots \mathrm{X}}=3 r_{\mathrm{HX}}=\frac{3}{2} r_{\mathrm{H} \ldots \mathrm{X}}
$$

Модифицируем условие (52) с помощью последнего соотношения:

$$
E_{\mathrm{H} . . . \mathrm{X}} \geq \frac{1}{12} r_{\mathrm{H} . . . \mathrm{X}}^{-2},
$$

что вместе с $\lambda=0, z=1$ даст неравенство для безразмерной энергии (30):

$$
y \geq y_{\min }=\frac{1}{6}
$$

Подчеркнем важный вывод из (55), сделанный в [20], об ограничении снизу для абсолютной величины энергии Н-связи. Это свойство позволяет выделить явление водородной связи среди других межмолекулярных сил, способных быть сколь угодно слабыми.

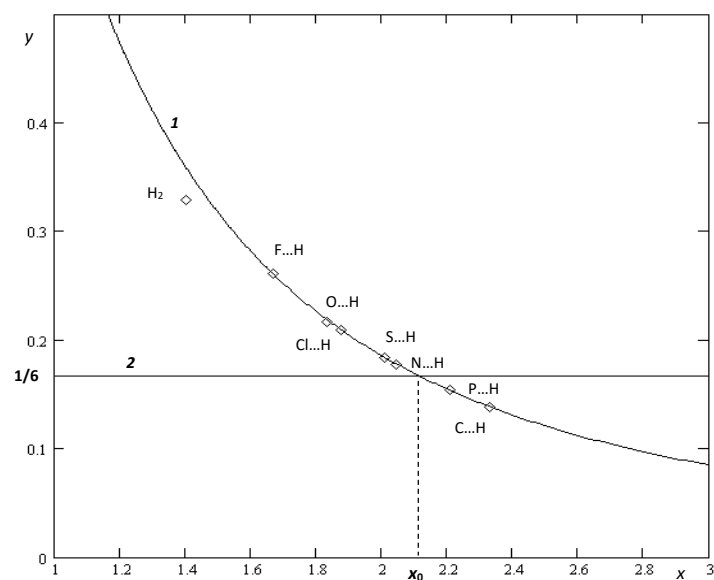

Рис. 2. График функции $y(x)$ (линия 1 ) по уравнению (28) с нанесенными характеристиками молекулы водорода и Н-связей элементов 2-го и 3-го периодов. Горизонтальная линия 2 соответствует нижнему пределу для ординаты водородной связи в чистом веществе (55). 
Итак, с одной стороны, характеристики Н-связей лежат на универсальной кривой электронной связи, а с другой - не могут быть ниже нижнего предела. График уравнения (28), построенный для области Н-связи (рис.2), удобен для иллюстрации этой мысли.

Из этого рисунка видно, какие гидриды способны к образованию Н-связи, а какие нет. Эти выводы согласуются с эмпирическими наблюдениями, что подтверждает их правильность. Главный вывод, важный для модели ММВ, в том, что приведенные величины энергии Н-связи для разных элементов лежат вблизи нижней границы существования электронной связи и могут быть приблизительно приравнены к ней.

\section{Вероятность водородного связывания}

Из-за узкой направленности Н-связи ее образование между двумя молекулами, связанными другими, «широкоугольными», видами ММВ, происходит с некоторой вероятностью $n_{H 12}$. В таком случае, число Н-связей, создаваемых молекулой в однородной среде, в среднем равно:

$$
n_{H}=z n_{H 12},
$$

где $z$ - максимально возможное координационное число молекулы в сетке водородных связей.

Каждая из двух молекул, образующих Н-связь, имеет электроотрицательные атомы, которые по отношению к протону Н-связи являются донорными или акцепторными. Для образования Н-связи гидридная группа должна сблизиться с другой молекулой так, чтобы донорный атом, его водород и акцепторный атом последовательно располагались в узком конусе, построенном на оси гидрида. Способность молекулы к образованию Н-связи характеризуется двумя гидридными числами $\left(d_{H},-a_{H}\right)$, где $d_{H}-$ донорное, или положительное, гидридное число, $-a_{H}-$ акцепторное, или отрицательное, гидридное число. В литературе известны аналоги этих чисел, выражающие кислотные и основные свойства по отношению к Н-связи, определяемые эмпирически [21]. Донорные атомы практически всегда могут быть акцепторами - как кислород у воды или спиртов, однако к акцепторам относятся также и «основания» - электроотрицательные атомы, не связанные ковалентно с водородом. «Основное» число является суммой гидридных чисел: $-b_{H}=-a_{H}+d_{H}$. Гидридные числа нормированы таким образом, что вероятность образования одной Н-связи между двумя молекулами (с индексами «1» и «2») равна сумме их произведений:

$$
n_{H 12}=d_{H 1} a_{H 2}+a_{H 1} d_{H 2}
$$

Каждый из членов суммы (57) представляет собой произведение вероятностей соответствующих групп и атомов занять нужное положение на оси создаваемой связи. Можно сказать, что в этом случае система попадает в «потенциальный колодец». В работе [22] дан алгоритм, осуществляющий попадание донорного атома одной молекулы и акцепторного атома другой молекулы в центр взаимодействия (рис.3). Из предложенной там схемы следует выражение для гидридных чисел через размеры

атомов и молекул: числа $d_{H}^{2}$ и $a_{H}^{2}$ есть доля внешней поверхности молекулы, занимаемая соответствующими атомами. 


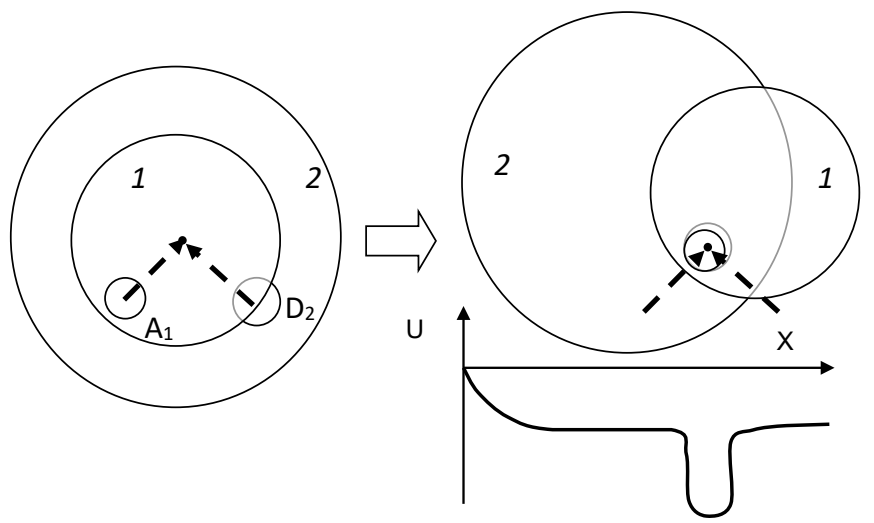

Рис. 3. Сечения молекул (1 и 2) с атомами ( $\mathrm{A}_{1}$ и $\left.\mathrm{D}_{2}\right)$, создающими Н-связь, и независимые движения молекул относительно центра дисперсионного взаимодействия (показано стрелками), которые требуется осуществить для попадания системы двух молекул в потенциальный колодец. График для потенциальной энергии, иллюстрирующий понятие потенциального колодца, дан внизу справа.

В распространенном случае квазиплоских молекул эти величины можно выразить через отношение площадей сечений соответствующих атомов $w_{a t, d}$ или $w_{a t, a}$ и их молекулы $w_{m o l}$ :

$$
d_{H}^{2}\left(a_{H}^{2}\right)=\frac{w_{a t, d(a)}}{2 w_{m o l}}
$$

Сечение молекулы (понимаемое как проекция молекулы на плоскость контакта молекул) различно в зависимости от угла наклона к плоскости контакта. В физической адсорбции усредненные проекции молекул на плоскость адсорбента, называемые молекулярными площадками, пропорциональны обобщенным зарядам (O3). В работах $[8,12,15,23]$ дано определение обобщенного заряда $Q$ по структурной формуле молекулы и формула для молекулярной площадки: $w=9.939 Q$, что позволяет связать гидридные числа со структурными свойствами:

$$
d_{H}^{2}\left(a_{H}^{2}\right)=\frac{Q_{d(a)}}{2 Q_{m o l}}
$$

Основываясь на этом определении, в работе [22] было выведено выражение для вероятности образования Н-связи между отдельной молекулой вещества «1» и молекулами жидкой фазы вещества «2»:

$$
n_{H 12}=\frac{\sqrt{Q_{a 1} Q_{d 2}}+\sqrt{Q_{d 1} Q_{a 2}}}{2\left(Q_{a 1} Q_{d 1}\right)^{1 / 4} \sqrt{Q_{\text {mol } 2}}}
$$

где $Q_{a}-$ O3 акцепторной группы, $Q_{d}-$ О3 донорной группы, $Q_{m o l}-$ О3 молекулы.

Случай взаимодействия между молекулой аналита и НФ в аналитической газовой хроматографии имеет свои особенности. В частности, образование более чем одной Н-связи из-за кратного роста энергии приводит к необратимой сорбции аналита, т.е. к его потере в колонке, поэтому в газовой хроматографии водородносвязываемые объекты характеризуются параметром $z=1$.

\section{Потенциальная энергия межмолекулярного взаимодействия}

Модель межмолекулярного взаимодействия (MMB) включает в себя неполярное ван-дер-ваальсово взаимодействие, полярные силы электрических диполей мо- 
лекул и водородные связи. Здесь рассматривается случай электрически нейтральных молекул в бесструктурной среде (газ, жидкость). Случай адсорбции молекулы «m» (с обобщенным зарядом $Q_{m}$ и дипольным моментом $\mu_{m}$ ) на аморфной неподвижной фазе «р», характеризующейся обобщенным зарядом $Q_{p}$ (или стандартной энергией $\left.U_{0}\right)$ и дипольным моментом $\mu_{p}$, с образованием $n_{H}$ водородных связей является частным по отношению к рассматриваемой модели ММВ.

Формула для потенциальной энергии ММВ включает в себя следующие 4 части [22,24]:

1. энергию неполярного взаимодействия между «m» и «p» (см. (22)):

$$
U(r)=Q_{m} Q_{p} u_{b}(r),
$$

где $u_{b}(r)=1.938 \cdot 10^{5} r^{-12}-5.115 r^{-6}=u_{b 0} f(r) \cdot\left(r_{b} / r\right)^{6}-$ функция межмолекулярного расстояния $r$ с минимумом в точке $r_{b}=6.505 \quad(0.3442 \quad$ нм), равным $u_{b 0} \equiv u_{b}\left(r_{b}\right)=-3.375 \cdot 10^{-5}$ (в молярных единицах -0.08854 кДж/моль), с безразмерной функцией $f(r)=2-\left(r / r_{b}\right)^{-6}=2-7.580 \cdot 10^{4} r^{-6}$. Для вычисления обобщенного заряда молекул часто достаточно использовать формулу:

$$
Q_{m o l}=\left(N_{\sigma}+N_{\pi} \sqrt{2}\right)^{3 / 4},
$$

где $N_{\sigma}, N_{\pi}$ - числа $\sigma$ - и $\pi$-электронов ковалентных связей атомов молекулы. На эти числа наложено условие: подсчитываемые валентные электроны удалены от места взаимодействия не дальше радиуса экранирования - величины, зависящей от их энергии. Радиус экранирования $r_{s}$ выражается через среднюю энергию валентного электрона $E_{l}$ (см. (27)):

$$
r_{s}=1.710 / \sqrt{E_{1}}
$$

В свою очередь, выражение для средней энергии валентного электрона вытекает из условия для средней плотности электронов, создающих О3:

$$
\bar{n} \propto N r_{s}^{-3} \propto N E_{1}^{3 / 2}=\sum_{i=1}^{N_{b}} z_{i}\left(E_{b i} / z_{i}\right)^{3 / 2},
$$

где $N_{b}=N_{\sigma} / 2$ - число ковалентных связей в молекуле, попадающих в сферу экранирования; $E_{b i}$ и $z_{i}$ - энергия и удвоенная кратность $i$-й ковалентной связи; $N=N_{\sigma}+N_{\pi}=\sum_{i=1}^{N_{b}} z_{i}$. Выражение (61) распространяется на случай адсорбции при замене обобщенного заряда стандартной энергией адсорбента: $U_{0}=Q_{p} u_{b 0}$.

Концепция экранирования валентных электронов, которая применяется в ТО3 приводит к характеристике плотной среды при ее взаимодействии с молекулой газовой фазы в виде О3 полусферы экранирования с центром, лежащим на поверхности (рис. 4a). В работе [25] выведена формула для обобщенного заряда плотной аморфной среды (например, жидкости) в качестве адсорбента, определенного как сумма О3 молекул в полусфере экранирования, примыкающей к поверхности:

$$
Q_{p}=\frac{\alpha \kappa}{2}\left(2 E_{1}\right)^{-3 / 2}
$$

где $\kappa$ - плотность укладки молекул жидкости ( 0.7 для малых молекул); $\alpha$ - коэффициент для О3 внутри сферы экранирования, равный 1 для линейных молекул. При описании жидкости как абсорбента (рис.4б) величина ОЗ удваивается по сравнению c (65); 


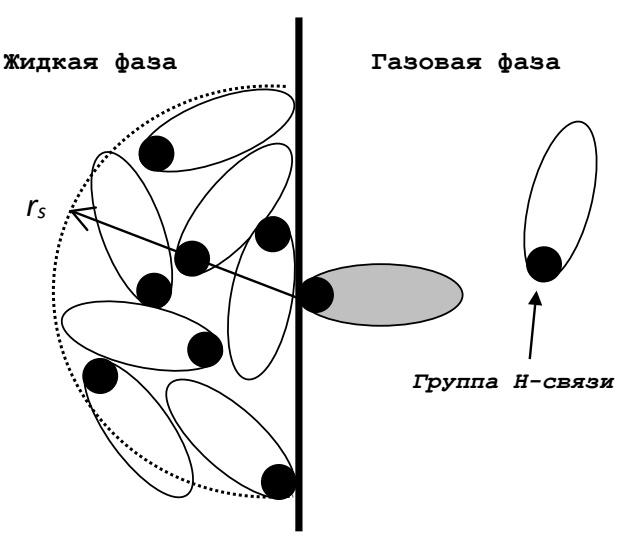

a

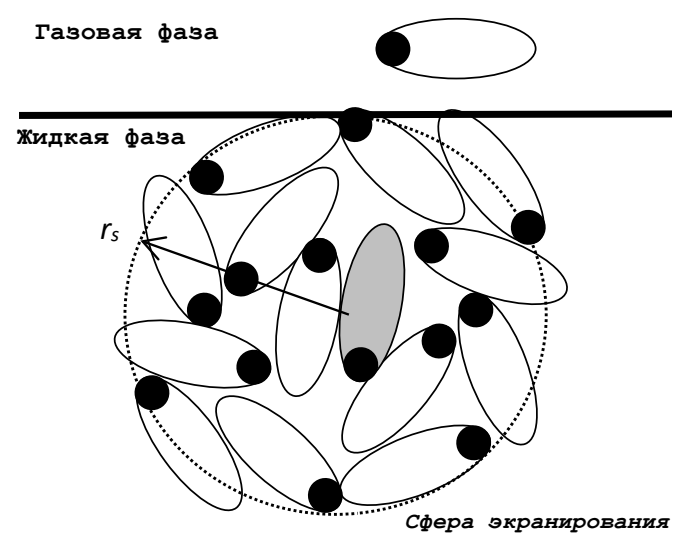

6

Рис. 4. Взаимодействие молекул пара и жидкости по механизмам адсорбции (а) и абсорбции (б). Показаны: поверхность раздела фаз (жирная линия) и сфера экранирования (пунктир), ограничивающая участие молекул жидкой фазы во взаимодействии с отдельно взятой молекулой (заштриховано).

2. энергию индукционного взаимодействия [5]:

$$
U_{\text {ind }}=-\left(\alpha_{p} \mu_{m}^{2}+\alpha_{m} \mu_{p}^{2}\right) r^{-6}
$$

где $\alpha$ - поляризуемость, связанная с обобщенным зарядом молекулы приближенной формулой $\alpha=a Q, a=4.948$ [12];

3. энергию ориентационного взаимодействия [5]:

$$
U_{o r}=-\frac{2 \mu_{m}^{2} \mu_{p}^{2}}{3 k_{B} T} r^{-6}
$$

где $k_{B}$ - постоянная Больцмана, $T$ - температура. Это выражение вытекает из формулы электростатики для взаимодействия двух диполей

$$
U_{\text {dip }}=\left[\left(\boldsymbol{\mu}_{\mathbf{1}} \boldsymbol{\mu}_{\mathbf{2}}\right)-3 r^{-2}\left(\boldsymbol{\mu}_{\mathbf{1}} \mathbf{r}\right)\left(\boldsymbol{\mu}_{\mathbf{2}} \mathbf{r}\right)\right] r^{-3},
$$

как среднестатистический эффект при произвольной ориентации диполей $\boldsymbol{\mu}_{1}$ и $\boldsymbol{\mu}_{2}$. Ориентационное взаимодействие сменяет диполь-дипольное при температурах, превышающих величину, необходимую для размораживания взаимного вращения связанных молекул;

4. энергию водородных связей (52) с учетом координационного числа и вероятности образования:

$$
E_{H} \approx 6 c_{H} n_{H} r^{-2}, c_{H}=\frac{1}{32}
$$

Сложив все вклады в предположении взаимной близости центров приложения сил, получим выражение общего вида:

$$
U_{m p}(r)=\left[U_{0}\left(Q_{m} f(r)+2 c \mu_{m}^{2}\right) r_{b}^{6}-a \mu_{p}^{2}\left(Q_{m}+c_{T} \mu_{m}^{2}\right)\right] r^{-6}-6 c_{H} n_{H} r^{-2}
$$

где введены коэффициенты: $c=0.9676, c_{T}=\frac{0.1347}{k_{B} T}$. Приравнивание производной функции (26) к нулю дает уравнение для расстояния $r=x r_{b}$ :

с коэффициентами:

$$
A x^{10}+B x^{6}-1=0
$$

$$
A=-\frac{c_{H} n_{H}}{U_{0} Q_{m} r_{b}^{2}}, B=1+c\left(\frac{\mu_{m}^{2}}{Q_{m}}+\frac{\mu_{p}^{2}}{Q_{p}}+c_{T} \frac{\mu_{m}^{2}}{Q_{m}} \frac{\mu_{p}^{2}}{Q_{p}}\right)
$$


Подстановка корня уравнения (71) $x_{e q}$ в виде $r_{e q}=r_{b} x_{e q}$ в (70) даст величину модуля приведенной равновесной энергии адсорбции (предполагается $A<<1 ; B-1<<1)$ :

$$
\varepsilon=6 A x_{e q}^{-2}+\left(2 B-x_{e q}^{-6}\right) x_{e q}^{-6} \approx(A+B)^{2}+4 A \approx 1+6 A+2(B-1),
$$

где ${ }_{\varepsilon} \equiv \frac{U_{m p}}{U_{0} Q_{m}} ; x_{e q} \equiv \frac{r_{e q}}{r_{b}} \approx(A+B)^{-1 / 6} ; r_{e q}$ - равновесное расстояние. Здесь можно заметить, что коэффициенты $A$ и $B$, а с ними и энергия зависят от величин вида $\mu^{2} / Q$, характеризующих полярность, и вида $n_{H} / Q$, характеризующих гидрофильность.

Выражение (73) применимо как для адсорбции, так и для общего случая MMB, поскольку адсорбент и молекула описываются аналогичными параметрами.

\section{Применение модели для расчета энтальпии парообразования во- ды, спиртов и кислот}

Вода и спирты. В гомологическом ряду воды и линейных одноатомных спиртов с общей формулой $\mathrm{C}_{\mathrm{n}} \mathrm{H}_{2 \mathrm{n}+1} \mathrm{OH} ; \mathrm{n}=0,1,2 \ldots$ обобщенный заряд согласно (62) подчиняется соотношению $Q=(6 n+4)^{3 / 4}$, куда подставлены электронные объемы углерода 4, водорода 1 и кислорода 2. Дипольные моменты 1.7 Д взяты для расчета спиртов и 1.85 Д - для воды. Ориентация молекул в жидкости произвольная, и поэтому образование Н-связи на ее поверхности имеет случайный характер. Такое поведение молекул жидкой воды и спиртов описывается формулой (60) для $n_{H 12}$, где задается $Q_{d}=3^{3 / 4}$ для гидроксила и $z=3$ :

$$
n_{H}=z n_{H 12}=3^{11 / 8} Q_{m o l}^{-1 / 2}
$$

В соответствии с (73) потенциальная энергия адсорбции молекулы воды или спирта на поверхности собственной жидкости описывается уравнением:

$$
-U_{m p} \approx 0.0885 Q_{m} Q_{p}+\frac{52.7}{\sqrt{Q_{m}}}+0.02653 \mu^{2}\left(Q_{p}+Q_{m}\right)+\frac{174.9}{T} \mu^{4} \text { кДж/моль }
$$

где в силу тождественности молекул пара и жидкости сделаны подстановки $Q_{\text {mol }} \equiv Q_{m}$ и $\mu_{p}^{2}=\mu_{m}^{2}=\mu^{2}$.

Обобщенный заряд поверхности жидкой фазы, состоящей из одинаковых молекул, определяется как О3 полусферы экранирования по формуле (65), которая для малых линейных молекул имеет вид: $0.35\left(2 E_{1}\right)^{-3 / 2}$. Однако, молекула, попавшая внутрь жидкости, взаимодействует с полной сферой экранирования, т.е. с удвоенной величиной:

$$
Q_{p} \approx 0.7\left(2 E_{1}\right)^{-3 / 2}
$$

Например, для воды половина энергии диссоциации ОН-связи $\mathrm{E}_{1}=248$ кДж/моль и согласно (76) $Q_{p}=7.78$. Для спирта (н-алкан-1-ол, $\mathrm{n} \geq 1$ - углеродное число) в формулу (64) подставлены: $N=6 \mathrm{n}+4$, энергии $3(\mathrm{n}-1)$ связей метиленового углерода $E_{b(-\mathrm{C}-)}=228$ кДж/моль, 3.5 связи метильного углерода $E_{b(\mathrm{C}-)}=$ 456 кДж/моль и 1.5 связи гидроксила $E_{b(\mathrm{O}-)}=428$ кДж/моль. Тогда получим: $E_{1} \approx 114 \cdot\left(\frac{3 \mathrm{n}+10.8}{3 \mathrm{n}+2}\right)^{2 / 3}$ кДж/моль и 


$$
Q_{p}=27.3\left(\frac{3 n+2}{3 n+10.8}\right)
$$

Из термодинамики известно, что при кипении любая молекула жидкости находится в состоянии молекулы на межфазной границе. Поэтому для правильности величины $z=3$, подставляемой в (74), следует рассматривать взаимодействие при температуре кипения жидкости.

Для расчета мольной энтальпии применили формулу:

$$
\Delta H \approx-U_{m p}+R T,
$$

где приближенное равенство записано для температур кипения при нормальном давлении. Расчетные значения для воды и спиртов являются ординатами точек $(+)$ на рис. 5.

Карбоновые кислоты.

В гомологическом ряду моноосновных карбоновых кислот с общей формулой $\mathrm{C}_{\mathrm{n}-1} \mathrm{H}_{2 \mathrm{n}-1} \mathrm{COOH} ; \mathrm{n}=1,2 \ldots$ обобщенный заряд отдельной молекулы, или «мономера», подчиняется соотношению $Q=(6 \mathrm{n}+2+2 \sqrt{2})^{3 / 4}$, куда подставлены вклады электронов углерода $\left(\mathrm{sp}^{3}\right) 4$, углерода $\left(\mathrm{sp}^{2}\right) 3+\sqrt{2}$, водорода 1 , кислорода гидроксильного 2 , кислорода карбонильного $1+\sqrt{2}$. Величина дипольного момента колеблется около 1.6 Д (1.60 \pm 0.15$)$.

При рассмотрении структуры жидкой карбоновой кислоты, можно видеть, что множество плотно расположенных мономеров состоит либо из отдельных циклических димеров, либо представляет собой водородно-связанную цепь мономеров, замыкаемую циклическим димером [22]. Ориентация молекул в жидкости произвольная, и поэтому образование Н-связи на поверхности имеет случайный характер. Координационное число при этом взаимодействии $z=1$. Такое поведение согласно формуле (60) описывается числом $n_{H}=1.686 Q_{m o l}^{-1 / 2}$. Циклические димеры, образующие паровую фазу кислот, имеют нулевой дипольный момент и отрицательное (т.е. акцепторное) гидридное число. Связь мономеров жесткая из-за циклического строения димера, поэтому О3 димера $\left(Q_{2 m}\right)$ создается удвоенным числом электронов по сравнению с мономером:

$$
Q_{2 m}=[2(6 n+4.83)]^{3 / 4}=2^{3 / 4} Q_{m}
$$

В соответствии с (73) при подстановке $\mu_{p}^{2} \approx \mu_{m}^{2}=0$ и $Q_{m o l} \equiv Q_{m}$ получим величину изменения потенциальной энергии димера при десорбции этого комплекса с поверхности своей жидкости:

$$
-U_{m p} \approx\left|u_{b 0}\right| Q_{p} Q_{2 m}+6 c_{H} n_{H} r_{b}^{-2} \approx 0.0885 Q_{2 m} Q_{p}+\frac{19.61}{\sqrt{Q_{m}}} \text { кДж/моль (80) }
$$

Аналогично (77) найдем величину О3 для сферы экранирования кислоты:

$$
Q_{p}=27.3\left(\frac{3 \mathrm{n}+2}{3 \mathrm{n}+11.3}\right)
$$

Результаты расчетов энергии десорбции молекулы с поверхности своей жидкости по формуле (80) и мольной энтальпии парообразования кислот по формуле (78): $\Delta H \approx-U_{m p}+R T-$ являются ординатами точек $(\times)$ на рис. 5. Уточним, что все величины приведены на моль димера $(\mathrm{RCOOH})_{2}$.

На рис. 5 приведен график с точками, абсциссы которых соответствуют экспериментальным значениям теплоты испарения жидкостей, а ординаты - теоретическим величинам, полученным для воды, гомологических рядов нормальных спиртов и нормальных карбоновых кислот. 


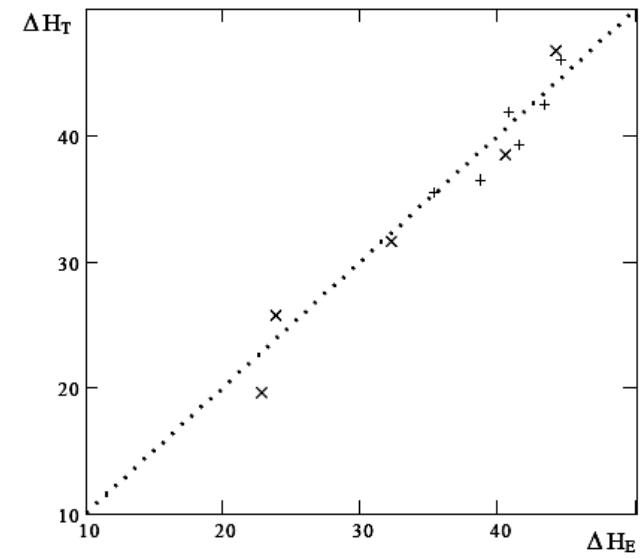

Рис. 5. Сравнение с экспериментальными величинами $\left(\Delta H_{E}\right)$ результатов расчета $\left(\Delta H_{T}\right)$ энтальпии парообразования воды (кДж/моль), спиртов и карбоновых кислот. Обозначения: (+) - вода и спирты, (×) - карбоновые кислоты.

Для проверки модели в работе [22] были проведены также достаточно точные априорные расчеты энергии диссоциации димеров рассмотренных молекул. Экспериментальные данные были взяты из оригинальных статей [26-30] и справочника [31]. Удовлетворительное соответствие расчетных и экспериментальных данных указывает на корректность развиваемой модели межмолекулярного взаимодействия.

\section{Заключение}

Таким образом, предложенный подход к описанию межмолекулярного взаимодействия общего типа дает достаточно хорошую оценку энергии сорбции молекулы на жидкой фазе, что позволит применить его при расчете хроматографического удерживания веществ по структурной формуле, задавая структуру фаз или их параметры полярности и гидрофильности.

Работа выполнена при финансовой поддержке РФФИ (грант 18-03-00382а).

\section{Список литературы}

1. Долгоносов А.М., Прудковский А.Г. // Журн. аналит. химии. 2002. Т.57. № 12. С. 1276-1283.

2. Долгоносов А.М., Прудковский А.Г., Колотилина Н.К. // Журн. аналит. химии. 2007. Т. 62. № 11. С. 1162-1171.

3. Долгоносов А. М., Прудковский А.Г. // Доклады Академии наук. 2013. Т. 449. № 3. C. 295-299.

4. Цирельсон В.Г. Квантовая химия. Молекулы, молекулярные системы и твердые тела. М. БИНОМ. 2012. 496 с.

5. Каплан И.Г. Межмолекулярные взаимодействия. Физическая интерпретация, компьютерные расчеты и модельные потенциалы. М. БИНОМ. Лаборатория знаний. 2014. 394 c.
6. Hobza P. // Annual Rep. Prog. Chem. Sect. 2004. Vol. 100. 3 p.

7. Долгоносов А.М. // Журн. физ.химии. 2001. Vol. 75. No 10. pp. 1813-1820.

8. Долгоносов А.М., Прудковский А.Г., Руденко Б.А. / В сб. «100 лет хроматографии» / Под ред. Б.А. Руденко. М. Наука. 2003. С. 269-310.

9. Ландау Л.Д., Лифшиц Е.М. Теоретическая физика. Т.3. Квантовая механика. М. ФИЗМАТЛИТ. 2004. 800 с.

10. Теория неоднородного электронного газа. / Ред. С.С. Лундквист, Н. Марч. М. Мир. 1987.400 c.

11. Koch W., Holthausen M.C., A Chemist's Guide to Density Functional Theory. Second Edition, 2001, Wiley-VCH Verlag GmbH, 293 p. 
12. Долгоносов А.М. Модель электронного газа и теория обобщенных зарядов для описания межатомных сил и адсорбции. М. Книжный дом ЛИБРОКОМ. 2009. 176 с.

13. Mott N.F. // Proc. Camb. Phil. Soc., 1936, Vol. 32, pp. 281-290.

14. Долгоносов А.М. // Журн. физ. химии. 2008. Т.82. № 12. С. 2306-2311.

15. Долгоносов А.М. // Журн. физ. химии. 2002. Т.76. № 12. С. 2216-2220.

16. Долгоносов А.М. // Журн. неорган. химии. 2017. Т.62. № 3. С. 330-336.

17. Долгоносов А.М. // Журн. неорг. химии. 2015. Т.60. № 2. С. 233-237.

18. Молекулярные постоянные неорганических соединений: Справочник / Под ред. Краснова К. С. Л. Химия. 1979. 448 с.

19. Долгоносов А.М. // Журн. неорган. химии. 2019. Т. 64. № 4. С. 389-396. DOI: 10.1134/S0044457X19040093.

20. Долгоносов А.М. // Журн. структ. химuи. 2019. Т. 60. № 11. С. 1765-1774. DOI: 10.26902/JSC id46400.

21. Раевский О.А. // Усn. химии. 1999. Т. 68. № 6. С. 555-576.
22. Долгоносов А.М. // Журн. структ. химии. 2020. Т. 61. № 7. С. 1107-1120.

23. Долгоносов А.М., Прудковский А.Г. // Журн. физ.химии. 2008. Т. 82. № 5. С.931939.

24. Зайцева Е.А., Долгоносов А.М. // Сорбционные и хроматографические проueccbl. 2019. T. 19. № 5. C. 525-541. DOI 10.17308/sorpchrom.2019.19/1167

25. Долгоносов А.М. // Изв.АН. Сер.хим. 2016. № 4. С. 952-963.

26. Соколов Н.Д. // Усn. физ. наук. 1955. Т. 57. C. 205-278.

27. Curtiss L.A., Frurip D.J., Blander M. // J. Chem. Phys. 1979. Vol. 71. pp. 2703-2711.

28. Provencal R.A., Casaes R.N., Roth K. et al. // J. Phys. Chem. A. 2000. Vol. 104. pp. 1423-1429

29. Claguet, A.D.H., Bernstein H.J. // Spectrochim. Acta, 1969, Vol. 25A, pp. 593-596.

30. Kollipost F., Wugt Larsen R., Domanskaya A.V., Nörenberg M153101.

31. CRC Handbook of Chemistry and Physics, Ed. D.R. Lide, Internet Version, http://www.hbcpnetbase.com, CRC Press, Boca

\title{
A model for a general type of intermolecular interaction between a molecule and a liquid phase based on the theory of generalized charges
}

\author{
(C) 2020 Dolgonosov A.M. \\ Vernadsky Institute of Geochemistry and Analytical Chemistry of Russian Academy of Sciences of the Rus- \\ sian Academy of Sciences, Moscow, Russia
}

The described study was conducted within the framework of the problem of modelling high performance chromatography methods. The study was aimed at explaining the aspects of the theory of intermolecular forces that are relevant to the description of the selectivity of the chromatographic stationary phases and, as a result, to the a priori calculation of the chromatographic retention. The article presents a brief overview of the theory of generalized charges and its applications for the description of interatomic bonds (a homopolar bond and a donor-acceptor bond) and intermolecular forces (ab initio Lennard-Jones potential). A hydrogen bond is described as an example of a hydride bond without electron exchange and the corresponding charge separation. Each aspect of the theory was tested in the experiments whose results are presented in the referenced works and, to some extend, in this article.

According to the suggested model, the energy of the intermolecular forces is determined by three different types of values, describing non-polar, polar, and hydrogen bonds. Each force is represented by its molecular descriptor: a generalised charge, a dipole moment, and two particles, which reflect the ability of the molecule to be either a donor, or acceptor in a hydrogen bond, respectively. The theory of generalised chargers helped us to obtain "structure-feature" bonds for all the elements of the intermolecular interaction energy. In particular, the article describes the contribution of the hydrogen bond to the overall energy of intermolecular interaction as the product of the threshold value, which has quantum-mechanical nature, and the probability of correct arrangement of the interacting molecules. This probability is determined as the probability of the system of interacting molecules being confined to a narrow potential well and is described by the parameters of the molecular structure. The suggested model was successfully used for a prior assessment of the heat of vaporisation of water, spirits, and acids, which proves the effectiveness of the developed approach.

Keywords: intermolecular forces, hydrogen bond, adsorption energy, heat of vaporisation, generalised charge. 


\section{References}

1. Dolgonosov A.M., Prudkovskiy A.G., J. Anal. Chem., 2002, Vol. 57, pp. 1089-1096.

2. Dolgonosov A.M., Prudkovskiy A.G., Kolotilina N.K., J. Anal. Chem. 2007, Vol. 62, pp. 1046-1054.

3. Dolgonosov A.M., Prudkovskiy A.G., Doklady Chem., 2013, Vol. 449, Part 1, pp. 8993.

4. Tsirel'son V.G. Kvantovaya khimiya. Molekuly, molekulyarnye sistemy I tverdye tela, M., BINOM, 2012, 496 p.

5. Kaplan I.G., Intermolecular Interactions: Physical Picture, Computational Methods, and Model Potentials. Wiley, 2006, 394 p.

6. Hobza P., Annual Rep. Prog. Chem. Sect., 2004, Vol. 100, pp. 3-10.

7. Dolgonosov A.M., Rus. J. Phys. Chem., 2001, Vol. 75, pp. 1659-1666.

8. Dolgonosov A.M., Prudkovskiy A.G., Rudenko B.A., 100 let khromatografii, Ed.: B.A. Rudenko, M., Nauka, 2003, pp. 269-310.

9. Landau L.D., Lifshitz E.M., Quantum Mechanics, 3rd Edition, Non-Relativistic Theory. Butterworth-Heinemann, 1981. 689 p.

10. Theory of the inhomogeneous electron gas, Ed.: S. Lundqvist and N.H. March. New York, Plenum Press, 1983. 390 p.

11. Koch W., Holthausen M. C., A Chemist's Guide to Density Functional Theory. Second Edition, 2001, Wiley-VCH Verlag GmbH, $293 p$.

12. Dolgonosov A.M., Model' elektronnogo gaza i teoriya obobschennykh zaryadov dlya opisaniya mezh'atomnyh sil I adsorbtsii. (Model of Electron Gas and Theory of Generalized Charges for Describing Inter-Atomic Forces and Adsorption (Rus.)). M., LIBROKOM, 2009, $176 \mathrm{p}$.

13. Mott N.F., Proc. Camb. Phil. Soc., 1936, Vol. 32, pp. 281-290.

14. Dolgonosov A.M., Russian Journal of Physical Chemistry A, 2008, Vol. 82, No 12, pp. 2079-2084.

\footnotetext{
Долгоносов Анатолий Михайлович - д.Х.н., ведущий научный сотрудник, лаборатория сорбционных методов, Институт геохимии и аналитической химии им. В.И. Вернадского РАН (ГЕОХИ РАН), Москва
}

15. Dolgonosov A.M., Rus. J. Phys. Chem., 2002, Vol.76, pp. 1659-1666

16. Dolgonosov A.M., Rus. J. Inorg. Chem., 2017, Vol. 62, pp. 344-350.

17. Dolgonosov A.M., Rus. J. Inorg. Chem., 2015, Vol. 60, pp. 194-197.

18. Molekulyarnye postoyannye neorganicheskikh soedineniy: Spravochnik, Ed.: Krasnov K.S. Leningrad, Khimiya. 1979, 448 p.

19. Dolgonosov A.M., Rus. J. Inorg. Chem., 2019, Vol. 64, pp.488-495.

20. Dolgonosov A.M., J. Struct. Chem., 2019, Vol. 60, pp. 1693-1702.

21. Raevskiy O.A., Russian Chemical Reviews, 1999, Vol. 68, pp. 505-524.

22. Dolgonosov A.M., J. Struct. Chem., 2020, Vol. 61, pp. 1107-1120.

23. Dolgonosov A.M., Prudkovskiy A.G., Rus. J. Phys. Chem., 2008, Vol. 82, pp. 812820.

24. Zaitceva E.A., Dolgonosov A.M., Sorptsionnye i khromatograficheskie protsessy, 2019, Vol. 19, pp. 525-541.

25. Dolgonosov A.M., Rus. Chem. Bul., 2016, Vol. 65, pp. 952-963.

26. Sokolov N.D., Uspekhi Fizicheskikh Nauk (Soviet Physics-Uspekhi), 1955, Vol. 57, pp. 205-278.

27. Curtiss L.A., Frurip D.J., Blander M., J. Chem. Phys., 1979, Vol. 71, pp. 2703-2711.

28. Provencal R.A., Casaes R.N., Roth K.et al., J. Phys. Chem. A, 2000, Vol. 104, pp. 14231429.

29. Claguet A.D.H., Bernstein H.J., Spectrochim. Acta, 1969, 25A, 593 p.

30. Kollipost F., Wugt Larsen R., Domanskaya A.V., Nörenberg M., Suhm M.A., J. Chem. Phys., 2012, Vol. 136, pp. 151101153101.

31. CRC Handbook of Chemistry and Physics, Ed. D.R. Lide, Internet Version, http://www.hbcpnetbase.com, CRC Press, Boca Raton, FL, 2005

Dolgonosov Anatoly M. - Dr.Sci.(Chem.), Leading scientific researcher, Lab of Sorption Methods, Vernadsky Institute of Geochemistry and Analytical Chemistry Russian Academy of Sciences (GEOKHI RAS), Moscow, amdolgo@mail.ru 